\title{
Le « bruit visuel » dans les Sermons sur Aggée de Savonarole
}

Il 'rumore visivo' nelle Prediche su Aggeo di Savonarola

The 'Visual Noise' in the Savonarolian Sermons On Haggai

Jean-Marc Rivière

\section{OpenEdition}

\section{Journals}

Édition électronique

URL : http://journals.openedition.org/cei/6105

DOI : 10.4000/cei.6105

ISSN : 2260-779X

\section{Éditeur}

UGA Éditions/Université Grenoble Alpes

Édition imprimée

ISBN : 978-2-37747-159-1

ISSN : $1770-9571$

Référence électronique

Jean-Marc Rivière, «Le « bruit visuel » dans les Sermons sur Aggée de Savonarole », Cahiers d'études italiennes [En ligne], 29 | 2019, mis en ligne le 30 septembre 2019, consulté le 28 mars 2021. URL http://journals.openedition.org/cei/6105; DOI : https://doi.org/10.4000/cei.6105

Ce document a été généré automatiquement le 28 mars 2021

(c) ELLUG 


\title{
Le « bruit visuel » dans les Sermons sur Aggée de Savonarole
}

\author{
Il 'rumore visivo' nelle Prediche su Aggeo di Savonarola \\ The 'Visual Noise' in the Savonarolian Sermons On Haggai
}

\author{
Jean-Marc Rivière
}

1 Parmi les questions majeures que soulève le moment savonarolien, il est celle de la performativité du discours: qu'un dominicain, qui n'est de surcroît même pas florentin, parvienne, en l'espace de quelques semaines, à établir son emprise sur une foule disparate, coupant transversalement des lignes de tensions qui avaient mis plusieurs générations à se cristalliser, relève d'une conjonction complexe de facteurs difficiles à percevoir dans l'ensemble de leurs interactions ${ }^{1}$. À la base de ce succès, il y a la rencontre, conjoncturelle et somme toute heureuse, d'un contexte et d'une personnalité, comme à chaque fois qu'un tel phénomène se produit. Dans le cas spécifique de Savonarole, ce constat préliminaire se heurte toutefois à un questionnement sous-jacent : selon quelles modalités un discours, conceptuellement et formellement fondé sur une analyse exégétique des saintes Écritures, bascule-t-il dans le champ politique? Ou, plus précisément : est-ce que ce glissement, du doctrinal au politique, profite de la fascination des fidèles et en est, dans ce cas, le prolongement naturel, ou bien, à l'inverse, en est-il l'un des points d'articulation systémiques ?

2 Pour nous, qui percevons ce phénomène de l'extérieur, de façon documentaire et dénuée de ce pathos qui faisait beaucoup au succès d'un prédicateur ${ }^{2}$, la question de la balance entre ces deux propositions est cruciale : mettant en jeu des mécanismes qui dépassent le simple champ pastoral, elle tisse en effet un réseau de causalité dont la brutalité de la chute de Savonarole semble être le point d'aboutissement. Dès les derniers mois de 1497, en effet, son discours semble perdre toute efficace et ne parvient pas à enrayer le phénomène de déconnexion qui lui fait perdre les faveurs d'une frange notable de ses concitoyens ${ }^{3}$.

3 À ce faisceau d'interrogations, il nous semble possible de répondre en étudiant la manière dont le discours savonarolien, dès l'instant de sa mise en action et 
concomitamment à la mutation institutionnelle de novembre-décembre 1494, travaille le concept de vision et est, en retour, travaillé par celui-ci. Le $1^{\mathrm{er}}$ novembre 1494 , en ouverture du premier sermon consacré au cycle d'Aggée, Savonarole déclare en effet :

Essendo, dilettissimi in Cristo Giesù, constituito da Dio l'uomo in questo mondo infra due termini constrarii l'uno all'altro, cioè tra il Paradiso e l'Inferno, ed essendo nel Paradiso ogni bene e nell'Inferno ogni male, doverebbe l'uomo, alzando l'occhio della mente, cercare con ogni sua sollicitudine volere sempre camminare con le buone sue operazioni verso el Paradiso, dove può sperare ogni bene, più tosto che col mal fare volere cercare l'Inferno, dove ha da temere di trovar ogni male. E perché sempre el mezzo participa della natura degli estremi, però molti si veggano accostarsi chi più e chi manco ad uno di questi estremi, cioè al paradiso o all'Inferno ${ }^{4}$.

Il est signifiant que l'expression «l'occhio della mente » et le verbe «vedere » soient ici associés, par un lien de proximité textuelle dont les connecteurs logiques soulignent qu'il n'est pas fortuit. On observe en effet, dans les Sermons sur Aggée, une saturation du champ pastoral par le lexique lié à la vision, dont l'usage récurrent et répétitif du verbe vedere (employé tant à la première personne qu'au pluriel, tant au présent, au passé, au futur qu'à l'impératif) est le signe le plus évident.

Il est tentant, en première analyse, de mettre l'accent sur la portée rhétorique d'une telle caractéristique discursive. Ainsi, dans son Institution oratoire (VI, 2.29), Quintilien nomme-t-il visio ${ }^{5}$ l'artifice grâce auquel, pour employer les mots de Jacqueline Lichtenstein, nous pouvons « nous représenter les images des choses absentes au point que nous ayons l'impression de les voir de nos propres yeux et de les tenir devant nous $^{6} »$. Par ailleurs, une analyse systématique du cycle sur Aggée montrerait combien l'effet de scansion entraîné par la reprise anaphorique du verbe vedere, combiné à l'effet de variatio induit par ses diverses formes morphologiques, se révèle efficace si l'on se place sur la stricte ligne de la performativité rhétorique.

6 Il nous semble toutefois que quelque chose de plus essentiel se noue ici autour du rapport à l'image, que l'on considérera ici dans l'acception large, susceptible de couvrir tout le spectre de ses possibles extensions dans le champ religieux, qu'en donne William John Thomas Mitchell :

Une part du problème repose dans l'ambiguïté qui caractérise le mot image : il peut dénoter à la fois un objet physique (une peinture ou une sculpture), une entité mentale et imaginaire, une imago psychologique, le contenu visuel des rêves, des souvenirs ou de la perception. Le mot joue un rôle dans les arts visuels ou textuels : il désigne le contenu représenté d'un tableau ou la totalité de sa gestalt formelle (ce qu'Adrian Stokes appelle "l'image en forme »); mais également un motif verbal, une chose ou une qualité, une métaphore ou une autre "figure", voire la totalité formelle d'un texte en tant qu' « icône verbale »"

Que l'image, comme facteur d'intermédiation, soit l'un des éléments cruciaux dans la relation de l'homme au monde nous est signalé, déjà, par Aristote ${ }^{8}$, avant qu'Erwin Panofsky, dans son essai intitulé Le problème du style dans les arts plastiques, ne synthétise d'une phrase définitive cette intime proximité, soulignant que « "le rapport de l'œil au monde" est en réalité le rapport de l'âme au monde de l'œil ${ }^{9}$ ».

Dans un rapport à l'autre fondé sur la parole, tel que celui qui lie le prédicateur à son auditoire, ce lien essentiel avec la vision semble toutefois relever d'une hétérogénéité de nature. Pour venir à bout de ce paradoxe liminaire, ou du moins tirer le premier fil d'une explicitation, tournons-nous vers Louis Marin. Dans son ouvrage Détruire la peinture, ce dernier parle, à propos de ses peintres de prédilection (Nicolas Poussin et 
Philippe de Champaigne, notamment), de ce «bruit visuel » qui « fait que, dans l'image regardée ici maintenant, glisse une autre, une autre encore, qu'elle convoque », avant de continuer ainsi :

Mais il fallait transcrire cette rumeur, écrire, donc nécessairement emprunter des détours, des traverses, choisir des procédés, des instruments pour faire lire, rendre lisible ce bruit: digressions, anacoluthes, parataxes, asyndètes, tout un arsenal de ruses et de pièges pour qu'un discours traverse de la peinture, pour que des tableaux traversent du langage, mais aussi des positions de discours et de savoir décalées, rompues, non immédiatement cohérentes ${ }^{10}$.

Notre réflexion est en réalité symétrique à celle de Louis Marin : là où celui-ci commente une peinture, celle $d u x{ }^{e}{ }^{e}$ français, qui vise à rendre visible " un bruit » - c'est-à-dire à faire la synthèse synesthésique de réalités sensorielles apparemment incompatibles ${ }^{11}-$, le défi qu'affronte Savonarole est de rendre audible ce qui relève d'un champ de perception distinct, celui de la vision. De transformer, donc, des mots en images ${ }^{12}$, au moyen de certains de ces "ruses » et "pièges » dont parle Louis Marin, qu'on nommera ici, par pudeur lexicale, « instruments ", " outils » ou " techniques », et qui relèvent d'une rhétorique verbale autant que figurative.

Savonarole utilise le lexique relevant de la vision pour marquer, en premier lieu, un constat : celui que la majorité de ses concitoyens sont plus portés vers le mal que vers le bien, et qu'ils se dirigent donc vers l'Enfer plutôt que vers le Paradis. Ainsi déclare-t-il, dans le sermon liminaire $\mathrm{du} 1^{\mathrm{er}}$ novembre :

Ma oimè, perchè ei si vede la maggiore parte delle persone declinare più verso l'Inferno che tendere verso el Paradiso, quanto è da dolersi della insensata creatura umana, che, posta intra el bene e il male e fatta libera di potere eleggere quale lei vuole, più presto cerchi la sua ruina che la sua felicità eterna, ingannando sè stessa per un poco di vanità di questo mondo.

[...] Né è da maravigliarsi del poco numero de' salvati, perché, considerata la bassezza dell'uomo, è pur gran cosa che Dio lo rilievi a tanta gloria; pochi si veggono che faccino penitenzia, e se alcuni la fanno, è poca el più delle volte, ed etiam spesso non è vera penitenzia, ma molte volte simulata ${ }^{13}$.

11 Dès ces premiers mots, Savonarole commence à construire ce qui sera l'une des problématiques majeures de son discours jusqu'au début de l'année 1495, à savoir la définition des contours d'appartenance au cercle de ses partisans, selon une partition entre amis, ennemis et tiepidi qui se dessine et se précise progressivement tout au long du cycle sur Aggée, parallèlement à la réflexion institutionnelle en cours ${ }^{14}$. La vision, à ce stade, est un acte concret, sensible, qui aboutit à un constat simple : ce que voit l'œil est, par nature, conforme à la réalité, donc à la vérité.

Dans un second temps, il s'agit pour Savonarole, à partir d'un commentaire exégétique des Écritures, de construire un transfert du monde sensible vers le monde céleste. Il emploie pour ce faire deux outils, la parabole et l'exemplum, dont la caractéristique commune est d'ouvrir sur un parcours analogique simple. Lorsque, le 9 novembre, Savonarole commente longuement une parabole centrée sur Achab et Jézabel, il utilise le substantif «documento » pour désigner celle-ci ${ }^{15}$. Or, en latin, un documentum est un exemple ou un modèle valant démonstration. Lorsque ce mot entre en italien, vers le milieu du XIV ${ }^{e}$ siècle, il désigne un document écrit qui a valeur de preuve. Savonarole utilise donc là ce terme dans une acception notariale : la vision garantit la véracité de ce qui est, et, par extension, de ce qui est dit lorsqu'on décrit ce qui est. 

autour du rapport à l'image. Dans sa brillante analyse du Timothée de Jan Van Eyck, Hans Belting a montré que la nouvelle conception du portrait, telle qu'elle se développe durant la seconde moitié $d u \mathrm{xv}^{\mathrm{e}}$ siècle, mais atteint sa pleine maturité en Italie justement à l'époque de Savonarole, repose sur un rapport d'étroite contiguïté entre l'image et la langue ${ }^{16}$, par lequel le portrait garantit la conformité - dans une acception large, qui s'étend jusqu'au domaine juridique - de ce qui est représenté par rapport à ce qui est. Et c'est le regard qui, en plaçant le portrait en position d'interface entre le sujet représenté et le spectateur ${ }^{17}$, fonde cette garantie de "loyale» ressemblance. Cette conformité presque juridique du discours avec la réalité, qu'offre la garantie du regard, c'est exactement celle qui pousse Machiavel à nommer «portraits» ses rapports diplomatiques sur la France et l'Allemagne, quelques années seulement après que s'exprime Savonarole ${ }^{18}$. Il y a là, dans des champs pourtant éloignés, une concomitance qui mérite d'être soulignée, à défaut de pouvoir être ici approfondie.

Concentrons-nous, à ce point, sur les ressorts à partir desquels se joue cette valeur documentaire de la vision dans le discours savonarolien. Dans le second sermon du cycle d'Aggée, en date du 2 novembre, Savonarole développe longuement l'exemplum d'une coupe en bois qui, en raison de sa matière, peine à retenir l'huile sainte qu'on y verse, tout comme l'âme du fidèle peine à retenir l'huile métaphorique de l'Esprit saint. Revenant sur l'arbre dont est tiré le bois dont est faite la coupe, il conclut ainsi son propos: «Verbigrazia, l'arbore è fatto per fare frutto, ma, quando tu vedi che uno arbore ̀̀ impedito e non fa el frutto tu lo tagli, chè è quivi indarno, non producendo frutto alcuno ${ }^{19}$. » Le verbe vedere sert ici à impliquer individuellement chaque auditeur par l'usage de la deuxième personne et par la forme d'adresse, tout en l'incitant à visualiser cet exemple concret à partir de son expérience sensible ${ }^{20}$.

Or, Savonarole poursuit ainsi : "Così se l'anima tua ti è data da Dio per fruttificare frutto centesimo per condurti in Paradiso con la grazia dello Spirito santo, e tu non lo fai, indarno $e$ invano hai ricevuta l'anima tua ${ }^{21}$. " La valeur structurante de l'adverbe liminaire dépasse ici le simple plan discursif : c'est bien parce qu'elle engendre un processus fondé sur la similitude que la vision permet la transition entre le monde sensible et le monde céleste. Elle fait office d'opérateur de conversion ${ }^{22}$, selon un processus assimilable à l'«opération de montage mental» décrite par Jérôme Baschet à propos de la monstration de l'image picturale du $\mathrm{Christ}^{23}$. Savonarole justifie d'ailleurs lui-même ce recours au principe de similitude dans son sermon du 13 décembre, lorsqu'il déclare : «Perché questo modo d'insegnare per parabole e similitudini è più delettabile; l'altra si è che meglio si tiene a mente quello che si dice e meglio si manda alla memoria ed è più connaturale all'uomo che vuole sapere, perché la scienzia ha principio dal senso ${ }^{24}$. » Il se place, de fait, dans la continuité de la similitudo per conlationem, telle qu'on la retrouve dans les traités antiques (par exemple dans la Rhétorique à Herennius), une pratique qui consiste, selon la formule classique, à « ante oculos ponere » les choses ${ }^{25}$.

Un tel transfert, de la dimension cognitive vers la dimension ontologique ${ }^{26}$, naît de ce que la vue est le plus noble des sens ${ }^{27}$, puisqu'elle ouvre sur la connaissance (comme d'ailleurs le veut le sens étymologique du verbe videre ${ }^{28}$. Savonarole se conforme là à la conception médiévale qui considère la vue, selon les mots de Thomas d'Aquin, comme «spiritualior et subtilior inter omnes sensus» (De anima, II, 1.14, n. 19) ${ }^{29}$. Cette supériorité naît de ce qu'elle est « maxime cognoscitivus » (Qucestiones perspicassimi peripatetici Ioannis de Ianduno in duodecim libros metaphysice, f. 6r), "magis cognoscitivus, quia est minus 
materialis » (Thomas d'Aquin, Summa theologice, I, q. 84, a. 3), et donc « secundum rationem cognitionis [...] primus» (Albert le Grand, Summa de creaturis, II, q. 19, a. 2) ${ }^{30}$. Le champ sémantique de la vision est ainsi intimement lié, dans les Sermons sur Aggée, à celui de la connaissance ${ }^{31}$. On est là dans la droite ligne de la pensée théologique qui, à cheval entre le bas Moyen Âge et le début de la Renaissance, a instauré une relation étroite, presque d'identification, entre voir et connaître, donc entre voir et croire ${ }^{32}$. Que la visio soit apprehensio, qu'elle soit liée à la vis cognoscitiva, on le lit par exemple dans la Somme théologique de Thomas d'Aquin ${ }^{33}$, chez Roger Bacon (The Opus Majus of Roger Bacon, II, 2) ou encore chez Albert le Grand qui, dans sa Summa de creaturis, rappelle ce que cette idée doit à Aristote.

La connaissance, c'est bien sûr avant tout celle que Dieu accorde à ses bienheureux, qui sont illuminés par sa lumière divine ${ }^{34}$ et auxquels il accorde le privilège de la visio beatifica ${ }^{35}$, héritée de la « visio Dei per essentiam ${ }^{36}$ » thomiste. Ainsi Savonarole déclare-til par exemple le 7 décembre :

[...] e fu concluso che noi dobbiamo ringraziare Dio, che ha scampato la città vostra questa volta dal pericolo in che elle è stata, il che tutti coloro che sono illuminati cognoscano, e sono inclinati a crederlo e confessare che tutto è stato fatto dalla mano di Dio; ed e converso chi non cognosce questo beneficio che abbiamo ricevuto da Dio, o egli è stolto o egli è cieco e ostinato nella sua mala volontà ${ }^{37}$.

Ceux qui sont capables de voir sont donc illuminés ${ }^{38}$ par la connaissance divine, en vertu de la théorie augustinienne qui assimile vision, connaissance et béatitude ${ }^{39}$. À l'inverse, ceux qui refusent ce don de Dieu sont plongés dans les «ténèbres des péchés ${ }^{40} »$ et restent donc aveugles, conformément au topos de la cécité ${ }^{41}$ largement usité par les prophètes vétéro-testamentaires.

De cette mise en tension entre la vision sensible et la vision considérée comme caractère de l'hypostase ${ }^{42}$ découle que le "faire voir » aboutit au «faire croire », c'està-dire, pour reprendre des catégories définies par les sémioticiens de l'image, que l'on passe de la visibilité à la figurabilitét3. En permettant le passage de l'historia (c'est-à-dire ce qu'on doit voir) à l'allegoria (c'est-à-dire ce qu'on doit croire), Savonarole ouvre à ses fidèles la voie de l'anagogia (c'est-à-dire l'élévation vers la béatitude éternelle ${ }^{44}$, dont il est en même temps l'origine, le vecteur et le médiateur.

Il crée par là même une seconde mise en tension, qui se noue entre la parole exégétique, fondée sur un discours de vérité, et la parole prophétique, qui s'articule autour d'un discours d'autorité. Pour Savonarole, il ne s'agit plus seulement de convaincre du bien-fondé de la vérité de la parole divine, voire du fait que l'émetteur du discours - c'est-à-dire lui-même - serait touché par une grâce prophétique qui légitimerait et validerait son propos. Il s'agit en réalité de convaincre de la possibilité d'un transfert de la parole évangélique vers la parole politique, c'est-à-dire de son retour, depuis le cadre pastoral, vers un cadre historique. Or, c'est là un balancement qui ne va pas de soi, puisqu'il va à l'encontre des modalités théoriques de l'acte prédicatoire.

21 Voir, c'est comprendre et, selon un lien de causalité inéluctable, c'est donc croire, en vertu du principe énoncé plus haut selon lequel la vision a un lien intime et nécessaire avec la vérité. Comme le dit Savonarole le $1^{\text {er }}$ novembre : «Tu sai, quando io ti dicevo: - Haec dicit Dominus, - tu non lo credevi; ora tu sei pur constretto a crederlo, perché tu lo vedi ${ }^{45}$. » La vision crée la foi en la parole divine, mais, parce qu'elle est portée, guidée et orientée par la parole du prédicateur, celui-ci acquiert à son tour, par métonymie 
- voire par contact ${ }^{46}$-, un statut prophétique, ce qu'exprime de la sorte Savonarole dans le sermon liminaire du cycle d'Aggée : «[...] e perché io te l'ho detto tanto tempo inanzi che le tribulazioni venissino e che Dio le manderà per purgare la Chiesa sua da tanti mali, però doveresti credere oramai, vedendone l'effetto ${ }^{47}$. "

Dans la continuité du pouvoir de vérité du discours exégétique, et presque par contamination, nous entrons désormais, de façon tout à fait fluide, dans un discours de vérité, qui fonde sa puissance sur la possibilité offerte, a posteriori, par la vision d'une vérification factuelle de la parole prophétique. Ainsi Savonarole n'hésite-t-il pas, le 30 novembre, à demander à son public: "Non hai tu veduto se egli è stato vero ${ }^{48}$ ?" Le prédicateur est, certes, un adjuvant du processus de vision et le porteur d'une parole prophétique ${ }^{49}$, mais il est aussi lui-même, simultanément, videns ${ }^{50}$, et donc capable de prévoir l'avenir ${ }^{51}$. Savonarole s'exclame ainsi, lors du sermon des 18-19 décembre : « $E$ io vi prometto che, se si fa questo, che sarete ricchissimi, potentissimi e gloriosissimi più che mai fusse Firenze. Questa è la vostra sicurtà: se voi non lo farete, io vi veggo esser in grandissimo pericolo ${ }^{52}$.»

Les derniers mots du cycle sur Aggée montrent combien la posture de prophète de Savonarole a gagné en autorité : "Se tu fai questo che io ti dico, e davvero, Firenze sarà in massima quiete. Altrimenti sarà il contrario, e tienlo bene a mente, e tutto sia a laude di Cristo, qui vivit e regnat in sæcula saeculorum. Amen ${ }^{53}$.» C'est en vertu de cette autorité qu'à partir du 7 novembre il commence à filer une métaphore, celle de l'Arche dans laquelle il invite ses fidèles à se rassembler, afin qu'ils se sauvent des turbulences à venir. Quelques mois plus tard, cette Arche accueillante deviendra une arche d'exclusion, d'où seront rejetés les ennemis et les tiepidi. Pour l'heure, il est intéressant d'observer que l'expression discursive de cette métaphore ouvre sur la dichotomie entre les «illuminati » et les «dannati, che sono privati della visione di Dio » et entrent, de ce fait, dans la catégorie des sots, des aveugles ou de ceux qui s'obstinent dans leurs errements ${ }^{54}$.

24 Aussitôt, toutefois, un transfert s'effectue vers une question nouvelle, celle de la temporalité : si « li beati ed el Cielo » nous dit Savonarole, «non invecchiano », car ils sont plongés dans l'Éternité céleste, «le cose temporali », en revanche, « mancano e invecchiano e però hanno bisogno di renovazione ». Le passage du plan céleste au plan sensible étant ainsi réalisé, il en conclut que "similmente la Chiesa di Cristo, che fu costrutta ed edificata dalla unione de' fedeli e delle loro buone operazioni, quando quelle mancano, si chiama invecchiata e ha bisogno di rinovarsi $i^{55}$ ». La métaphore de l'Arche mène donc, par l'intermédiaire de la vision, au discours politique. Ce même processus se vérifie à chaque fois que Savonarole a recours à la métaphore, qu'il fasse par exemple référence à la reconstruction du Temple de Jérusalem pour renvoyer à la nécessaire rénovation morale de la cité ${ }^{56}$, ou à ce marin perdu en mer qui, poussé par des vents contraires, ne voit aucun port, pour signifier combien cette rénovation se trouve confrontée à des forces hostiles ${ }^{57}$.

Tout comme la similitude, la métaphore fonctionne par rapprochement analogique, mais elle exige, pour se dévoiler, un engagement intellectuel plus grand ${ }^{58}$. Elle fonctionne en cela comme l'allégorie, dont Savonarole, le 13 décembre, alors qu'il s'apprête à se lancer dans l'explication de l'épisode d'Élysée et de la femme sunamite, tiré du Livre des Rois (II, 4:8-30), nous explique les fondements dans un long excursus théorique : 
Bisogna che quella scrittura che ha allegoria sia cosa ferma e non favole e ch'el suo senso litterale sia vero e saldo [...]. Non hanno allegoria né significato vero le favole de' poeti, come alcuni dicano ed errano grandemente. La Scrittura sacra ha allegoria, $[. .$.$] che così è stata vera in fatto, significhi ancora altre cose che di poi$ hanno a venire ${ }^{59}$.

L'allégorie, tout comme la métaphore, est donc un processus complexe de translation de sens. Or, l'effort que requiert son dévoilement, pour des fidèles qui ont été capables d'ouvrir l'« œil de leur esprit ", n'est pas insurmontable, surtout s'ils sont guidés dans ce cheminement intellectuel. Le retour à la réalité sensible est à ce prix.

Le discours politique savonarolien, en ces premiers mois, est encore relativement basique: le danger menace, et il faut en prendre conscience pour s'y préparer. C'est pourquoi, le 12 décembre, Savonarole intime cet ordre à la cité, avec l'autorité que lui confère son statut prophétique: "Apri gli occhi, ti dico io; apri gli occhi della ragione e vederai in che pericolo ti trovi ${ }^{60}$. $~ L a$ vision n'a donc pas une simple fonction théologique, mais elle a également aussi une claire valeur opératoire dans le tissu discursif pastoral. Et le vecteur de ce transfert, ce autour de quoi tourne tout le travail savonarolien sur la vision, ce sont justement ces "occhi della ragione ", qui rappellent "l'occhio della mente » cité en ouverture de notre propos. C'est là une expression qu'on trouve paradoxalement assez peu chez Savonarole : outre cette occurrence liminaire dans les Sermons sur Aggée, elle revient sous une forme légèrement différente, "l'occhio dello intelletto ${ }^{61} "$, dans le Trattato circa el reggimento e governo della città di Firenze (III, 2) ${ }^{62}$ et dans sa forme originelle, mais plurielle, "gli occhi della mente ${ }^{63}$ ", dans les Sermons sur les Psaumes. Elle n'en est pas moins intéressante, parce qu'elle relie Savonarole à Bernardin de Sienne (qui fait une distinction entre "l'occhio corporale » et "l'occhio mentale » ${ }^{64}$ ), mais aussi aux grands théologiens médiévaux, tel saint Bonaventure qui, dans les Meditaciones vitoe Christi qui lui sont traditionnellement attribuées, parle de "l'occhio della mente tu $a^{65} »$. Elle trace également une ligne de continuité entre Savonarole et la pensée antique, notamment celle de Quintilien qui, dans son De Institutione Oratoria $(8,3,62)$ définit l'« oculi mentis» comme la faculté de concevoir des images mentales ${ }^{66}$, ou plus précisément, comme l'indique Jean-Pierre Aygon, «la capacité de se représenter directement une image mentale, transmise par les mots, comme si elle l'était par la perception, sans médiation rationnelle ${ }^{67} \%$.

L'acte opératoire autour duquel s'articule l'ouverture de cet «œil de l'esprit » est toutefois complexe. Pour parvenir à voir, ne fût-ce que provisoirement, la vérité éternelle, il a besoin, tout comme le corps, de s'exercer par un apprentissage régulier et par une pratique assidue, comme l'indiquait déjà saint Augustin ${ }^{68}$. Savonarole confirme d'ailleurs cette nécessité, lorsqu'il déclare, le 3 novembre: "Piglia questo libro [del Crucifisso] [...] ; lessilo ogni giorno, e questo ti gioverà più che tutti gli studii del mondo. Impara di orare, impara a meditare e contemplare, ed eccita e innalza la mente tua ${ }^{69} . »$ La performativité des sermons savonaroliens s'explique ainsi en partie par l'intensité et la cyclicité de ces prêches, qui n'hésitent pas, de jour en jour, à revenir sur les mêmes images pour contraindre les fidèles à la concentration, pour les obliger à avoir, selon les mots de Savonarole, "la mente fissa a' sepolcri de' santi e alla fede che gli ha condotti in Paradiso $^{70} »$. Ce n'est que par ce processus complexe, fondé sur la récurrence et la remémoration ${ }^{71}$, que Savonarole peut amener ses fidèles, selon une fascinante expression oxymorique qu'on trouve dans l'un des sermons consacrés aux Psaumes (24 mai 1495), à " voir ce qu'il leur dit" ${ }^{72}$ ", en vertu d'un processus qu'il décrit ainsi : «Ora, a proposito nostro: la voce del predicatore va all'orecchio dell'audiente e l'anima la piglia e 
manda allo intelletto, che la consideri; se l'intelletto e l'anima è ben preparato, quella voce penetra, chè ha virtù penetrativa dello Spirito santo ${ }^{73}$. » Pour que s'ouvre l'œil de l'esprit, la voix du prédicateur est nécessaire, mais pas suffisante : il faut cette préparation, sans laquelle la parole glisse à travers l'oreille du fidèle et ne parvient pas à atteindre son âme.

Ainsi, si l'on retire à Savonarole la possibilité d'entraîner ses fidèles à ouvrir l'œil de leur esprit - notamment en l'empêchant matériellement de prêcher et en brisant cette nécessaire récurrence -, alors seule reste sa voix, désormais inefficiente à créer ce lien entre l'individualité et la collectivité ${ }^{74}$ qui joue un rôle structurant non seulement dans le discours pastoral mais, de façon plus générique, dans l'acte d'image lui-même ${ }^{75}$. Savonarole a alors beau " tellement crier et tellement vociférer ${ }^{76}$ ", cela ne suffit pas : il est inaudible. Le «champ social du visuel », comme le nomme W. J. T. Mitchell ${ }^{77}$, lui est désormais inaccessible, car le fil délicat qui le reliait à la foule des fidèles s'est brisé. Le lexique de la vision demeure, mais il perd toute efficace et court le risque de se voir réduit à un rôle de pur marqueur discursif. C'est là sans doute une hypothèse susceptible d'expliquer le décalage qu'on observe durant les dernières semaines de sa prédication, cette impression que son discours tourne à vide et n'atteint plus sa cible ${ }^{78}$.

\section{BIBLIOGRAPHIE}

ARASSE Daniel, « Ferevat pietate populus. Art, dévotion et société autour de la glorification de S. Bernardin de Sienne ", Mélanges de l'École française de Rome, t. 89, nº 1, 1977, p. 189-263.

AYGon Jean-Pierre, « "Les yeux de l'esprit” (oculi mentis, Quintilien, I. 0., 8, 3, 62) : la relation entre les images et la raison chez les rhéteurs et chez Sénèque ", Pallas, $n^{\circ} 93,2013$, disponible en ligne sur <https://journals.openedition.org/pallas/1501>.

BASCHET Jérôme, «Vision béatifique et représentations du Paradis $\left(\mathrm{XI}^{\mathrm{e}}-\mathrm{XV}^{\mathrm{e}}\right.$ siècle) ", Micrologus, vol. VI, La visione e lo sguardo nel Medio Evo, II, 1998, p. 73-93.

Belting Hans, Pour une anthropologie des images, Paris, Gallimard, 2004.

BERLIOZ Jacques, «Le récit efficace : l'exemplum au service de la prédication (XIII ${ }^{\mathrm{e}}-\mathrm{XV}^{\mathrm{e}}$ siècles », Mélanges de l'École française de Rome, t. 92, nº 1, 1980, p. 113-146.

BOCHET Isabelle, « Le statut de l'image dans la pensée augustinienne », Archives de philosophie, t. 72, 2009, p. 249-269.

Bolzoni Lina, La rete delle immagini. Predicazione in volgare dalle origini a Bernardino da Siena, Turin, Einaudi, 2009.

BREDEKAMP Horst, Théorie de l'acte d'image, Paris, Éditions de la Découverte, 2015.

Didi-Huberman Georges, Devant l'image, Paris, Les Éditions de Minuit, 1990.

Didi-HubERMAN Georges, Fra Angelico. Dissemblance et figuration, Paris, Flammarion, 1995.

Eco Umberto, Écrits sur la pensée au Moyen Âge, Paris, Grasset, 2016. 
IMBACH Ruedi et PUTALlaz François-Xavier, « Notes sur l'usage du terme imago chez Thomas d'Aquin », Micrologus, vol. 5, La visione e lo sguardo nel Medio Evo, I, 1997, p. 69-88.

MARIN Louis, Détruire la peinture, Paris, Flammarion, 1997.

MILES Margaret, « The Eye of the Body and the Eye of the Mind in Saint Augustine's De trinitate and Confessions ", The Journal of Religion, vol. 63, $\mathrm{n}^{\circ}$ 2, 1983, p. 125-142.

Mitchell W. J. T., « Que veulent réellement les images? », dans E. Alloa (éd.), Penser l'image, Dijon, Les Presses du Réel, 2010 et 2011, p. 211-247.

NELSON Robert S., « Descarte's Cow and Other Domestications of the Visual », dans R. Nelson (éd.), Visuality before and beyond the Renaissance. Seeing as Others Saw, Cambridge, Cambridge University Press, 2000, p. 1-20.

Niccoli Ottavia, Vedere con gli occhi del cuore. Alle origini del potere delle immagini, Rome-Bari, Laterza, 2011.

RIVIÈRE Jean-Marc, «Figuration et figurabilité dans le Portrait de Savonarole par Fra Bartolomeo », dans Y. Gouchan, J. Moulin et N. Phuong Ngoc (éds), La Vérité d'une vie. Études sur la véridiction en biographie, Paris, Champion, 2019, p. 305-317.

SAVONARola Girolamo, De Simplicitate vitee christiance, Rome, Belardetti, 1959.

SAVonARola Girolamo, Prediche sopra Aggeo, Rome, Belardetti, 1965.

Savonarola Girolamo, Commento al salmo Miserere mei, Deus, Milano, Glossa, 2012.

SPINOSA Giacinta, «Visione sensibile e intellettuale. Convergenze gnoseologiche e linguistiche nella semantica della visione medievale ", Micrologus, vol. V, La visione e lo sguardo nel Medio Evo, I, 1997, p. 119-134.

TORRELL Jean-Pierre, « La vision de Dieu per essentiam selon saint Thomas d'Aquin », Micrologus, vol. V, La visione e lo sguardo nel Medio Evo, I, 1997, p. 43-68.

WiLliamson Beth, « Sensory Experience in Medieval Devotion: Sound and Vision, Invisibility and Silence», Speculum, vol. 88, n 1, 2013, p. 1-43.

\section{NOTES}

1. Voir G. C. Garfagnini (éd.), Una città e il suo profeta. Firenze di fronte al Savonarola, Florence, Sismel-Edizioni del Galluzzo, 2001.

2. Cf. D. Arasse, "Ferevat pietate populus. Art, dévotion et société autour de la glorification de S. Bernardin de Sienne ", Mélanges de l'École française de Rome, t. 89, n 1, 1977, p. 189-263; V. Berardini, « Prédicateurs et acteurs. À la recherche d'indices de performance dans les sermons de la fin du Moyen Âge », dans M. Bouhaïk-Gironès et M. A. Polo de Beaulieu (dir.), Prédication et performance du XII au XVI e siècle, Paris, Garnier, 2013, p. 79-90.

3. Voir J.-M. Rivière, "La figure de l'ennemi dans le discours politique savonarolien ", dans (Dis)qualifier l'ennemi, Actes du colloque Université Paul Valéry Montpellier 3 (8-10 novembre 2017), à paraître. 4. G. Savonarola, Prediche sopra Aggeo, Rome, Belardetti, 1965, p. 1. L'italique est ici, comme dans les citations suivantes, de notre fait.

5. Cf. G. Spinosa, «Visione sensibile e intellettuale. Convergenze gnoseologiche e linguistiche nella semantica della visione medievale ", Micrologus, vol. V, La visione e lo sguardo nel Medio Evo, I, 1997, p. 119. 
6. J. Liechtenstein, La Couleur éloquente. Rhétorique et peinture à l'âge classique, Paris, Flammarion, 2013, p. 146.

7. W. J. T. Mitchell, Que veulent les images? Une critique de la raison visuelle, Dijon, Les Presses du Réel, 2014, p. 25.

8. "Quant à la pensée discursive de l'âme, les images lui tiennent lieu de sensation. Et quand l'objet est bon ou mauvais, elle affirme ou nie, fuit ou poursuit. C'est pourquoi l'âme ne pense jamais sans image. De son côté, l'âme douée de réflexion dispose de représentations qui tiennent lieu de sensations. Et quand un bien ou un mal se trouve énoncé ou nié, il y a également un mouvement de fuite ou de poursuite. Aussi l'âme ne pense-t-elle jamais sans représentation. » (Aristote, De l'âme (III, 7, 431a), Paris, Flammarion, 1993, p. 273)

9. E. Panofsky, «Le problème du style dans les arts plastiques (1915) », dans Id., La perspective comme forme symbolique et autres essais, Paris, Minuit, 1975, p. 188.

10. L. Marin, Détruire la peinture, Paris, Flammarion, 1997, p. 8.

11. Marin est d'ailleurs conscient de cette incompatibilité de principe, lorsqu'il indique que «donner à voir la voix, ce serait l'étrange entreprise, et peut-être le comble de l'entreprise, nommée représentation de peinture » (L. Marin, "Aux marges de la peinture : voir la voix », L'Écrit du temps, $\mathrm{n}^{\circ} 17,1988$, p. 62).

12. Bien que ce ne soit pas là l'objet direct de notre présente étude, soulignons l'importance, dans un tel processus, de l'iconographie contemporaine de Savonarole, étudiée notamment dans L. Sebregondi, Iconografia di Girolamo Savonarola 1495-1998, Florence, Sismel-Edizioni del Galluzzo, 2004.

13. G. Savonarola, Prediche sopra Aggeo, ouvr. cité, p. 1-3.

14. Voir J.-M. Rivière, «La figure de l'ennemi », art. cité.

15. G. Savonarola, Prediche sopra Aggeo, ouvr. cité, p. 67. Ce terme est employé, dans la même acception, lors des sermons des 9 et 16 novembre et du 8 décembre (ibid., p. 63-65, 78-79 et 146).

16. H. Belting, Miroir du monde. L'invention du tableau dans les Pays-Bas, Paris, Hazan, 2014, p. 62-69.

17. Id., Faces. Une histoire du visage, Paris, Gallimard, 2017, p. 164.

18. J.-M. Rivière, L'expérience de l'autre. Les premières missions diplomatiques de Machiavel, Guicciardini et Vettori: regards croisés sur la France, l'Allemagne et l'Espagne, Aix-en-Provence, Presses universitaires de Provence, 2018, p. 16-19.

19. G. Savonarola, Prediche sopra Aggeo, ouvr. cité, p. 29.

20. Ce rapport à la réalité sensible est fondamental dans l'efficace de l'exemplum puisque celui-ci, selon Jacques Le Goff, est une « anecdote présentée comme réellement arrivée, par laquelle le prédicateur cherche à convaincre son auditoire d'une réalité religieuse utile à son salut » (cité dans N. Bériou, "L'art de convaincre dans la prédication de Ranulphe d'Homblières ", dans Faire croire. Modalités de la diffusion et de la réception des messages religieux du XII au XV siècle, Actes de la table ronde de Rome (22-23 juin 1979), Rome, École française de Rome, 1981, p. 56-57).

21. G. Savonarola, Prediche sopra Aggeo, ouvr. cité, p. 29.

22. Cf. L. Bolzoni, La rete delle immagini. Predicazione in volgare dalle origini a Bernardino da Siena, Turin, Einaudi, 2009, p. 58.

23. J. Baschet, "Images en acte et agir social », dans A. Dierkens, G. Bartholeyns et T. Golsenne (éds), La performance des images, Bruxelles, Éditions de l'Université de Bruxelles, 2010, p. 13.

24. G. Savonarola, Prediche sopra Aggeo, ouvr. cité, p. 192.

25. Cf. F. Libral, «Entre similitudes et métaphores. Amplification et optique dans la prédication en France (v. 1600-1670)», Exercices de rhétorique, $\mathrm{n}^{\circ} 4,2014$, p. 1-6, disponible en ligne sur <https://journals.openedition.org/rhetorique/351> ; J. Berlioz, «Le récit efficace : l'exemplum au service de la prédication ( $\mathrm{XIII}^{\mathrm{e}}-\mathrm{XV}^{\mathrm{e}}$ siècles ", Mélanges de l'École française de Rome, t. $92, \mathrm{n}^{\circ} 1$, 1980, p. 113-146 ; J. Berlioz et M. A. Polo de Beaulieu, "Introduction générale », dans M. A. Polo de Beaulieu, P. Collomb et J. Berlioz (dir.), Le Tonnerre des exemples. Exempla et médiation culturelle dans l'Occident médiéval, Rennes, Presses universitaires de Rennes, 2010, p. 11-15. 
26. Cf. R. Imbach et F.-X. Putallaz, " Notes sur l'usage du terme imago chez Thomas d'Aquin », Micrologus, vol. V, La visione e lo sguardo nel Medio Evo, I, 1997, p. 73.

27. Cf. G. Savonarola, Prediche sopra Amos e Zaccharia, vol. 1, Rome, Belardetti, 1971-1972, p. 303. Comme on le lit dans une Sacra rappresentazione florentine du $\mathrm{Xv}^{\mathrm{e}}$ siècle, "l'ochio si dice ch'è la prima porta per la quale l'intellecto intende e gusta " (citée dans A. Prosperi, Giustizia bendata. Percorsi storici di un'immagine, Turin, Einaudi, 2008, p. 10).

28. Ainsi lit-on à l'entrée viso, -ere du Dictionnaire étymologique de la langue latine, que «le sens initial de videre était relatif à la connaissance, non à l'acte de "voir" ou d'"observer" [...]. Des trois racines qui servaient en indo-européen à indiquer la "vision", le latin ignore *derk-, qui indiquait proprement l'acte de voir et qui fournissait des aoristes et des parfaits [...] ; il a les deux autres, l'une dans specio, l'autre dans oculus et dans les composés des types ferox et antiquus; c'est la racine qui sert à indiquer l'organe et, au désidératif, l'acte de l'organe. De plus, il recourt à la racine *weid-, ou le sens de "voir» est un cas particulier d'un emploi plus général : *weidindique la vision en tant qu'elle sert à la connaissance. Le parfait de *weid-, qui exprime un résultat acquis, a le sens de "savoir" "(A. Ernout et A. Meillet, Dictionnaire étymologique de la langue latine, Paris, Klincksieck, 2001, p. 734).

29. Cf. O. Niccoli, Vedere con gli occhi del cuore. Alle origini del potere delle immagini, Rome-Bari, Laterza, 2011, p. 14.

30. Cf. G. Spinosa, « Visione sensibile », art. cité, p. 125-132.

31. Cf. G. Savonarola, Prediche sopra Aggeo, ouvr. cité, p. 10 et 390.

32. Cf. O. Niccoli, Vedere, ouvr. cité, p. 14.

33. U. Eco, Écrits sur la pensée au Moyen Âge, Paris, Grasset, 2016, p. 145-146.

34. Cf. G. Savonarola, Prediche sopra Aggeo, ouvr. cité, p. 5. Une excellente synthèse sur la manière dont la lumière devient, au Moyen Âge, la base métaphorique primordiale dans la quête spirituelle se trouve dans U. Eco, Arts et beauté dans l'esthétique médiévale, Paris, Grasset, 1997, p. 83-91.

35. Cf. O. Niccoli, Vedere, ouvr. cité, p. 14.

36. Cf. J.-P. Torrell, «La vision de Dieu per essentiam selon saint Thomas d'Aquin", Micrologus, vol. V, La visione e lo sguardo nel Medio Evo, I, 1997, p. 43-68; D. Arasse, «Extases et visions béatifiques à l'apogée de la Renaissance : quatre images de Raphaël ", Mélanges de l'École française de Rome, t. 84, $\mathrm{n}^{\circ}$ 2, 1972, p. 403.

37. G. Savonarola, Prediche sopra Aggeo, ouvr. cité, p. 123-124.

38. Cf. L. Marin, « L'efficace », art. cité, p. 106.

39. Cf. J. Baschet, "Vision béatifique et représentations du Paradis ( $\mathrm{XI}^{\mathrm{e}}-\mathrm{XV}^{\mathrm{e}}$ siècle) », Micrologus, vol. VI, La visione e lo sguardo nel Medio Evo, II, 1998, p. 74 ; I. Bochet, « Le statut de l'image dans la pensée augustinienne ", Archives de philosophie, t. 72, 2009, p. 249-269.

40. G. Savonarola, Prediche sopra Aggeo, ouvr. cité, p. 27. Cette idée est exprimée à d'autres reprises, notamment le 23 novembre (ibid., p. 92).

41. On retrouve à plusieurs reprises ce topos de la cécité chez Savonarole, notamment dans les sermons des $1^{\text {er }}$ et 30 novembre, ainsi que du 7 décembre (ibid., p. 17, 111 et 128).

42. Dans son De Simplicitate vitce christiance, publié en latin le 28 août 1496, puis en langue vulgaire le 31 octobre de la même année, Savonarole note que «el fine del cristiano è quello che non si vede » (G. Savonarola, De Simplicitate vitce christiance, Rome, Belardetti, 1959, p. 160).

43. Cf. J. Liechtenstein, La Couleur éloquente, ouvr. cité, p. 139 ; G. Didi-Huberman, Devant l'image, Paris, Les Éditions de Minuit, 1990, p. 38.

44. Cf. G. Didi-Huberman, Fra Angelico. Dissemblance et figuration, Paris, Flammarion, 1995, p. 65-66.

45. G. Savonarola, Prediche sopra Aggeo, ouvr. cité, p. 12.

46. Il ne nous semble pas trop audacieux de tisser un lien entre ce processus et l'importance du contact comme fondement de la ressemblance, selon les modalités décrites dans G. Didi- 
Huberman, La ressemblance par contact. Archéologie, anachronisme et modernité de l'empreinte, Paris, Éditions de Minuit, 2008.

47. G. Savonarola, Prediche sopra Aggeo, ouvr. cité, p. 10.

48. Ibid., p. 110.

49. Ainsi Savonarole déclare-t-il lors du sermon du 30 novembre : «[...] e' segni, che tu n'hai, da dovere ricognoscere questo beneficio e che dimostrano che Dio ti vuol bene, Firenze, io te li mostro. » (Ibid., p. 111)

50. Cf. A. Ernout et A. Meillet, Dictionnaire étymologique, ouvr. cité, p. 733.

51. Notons que le prophète, par un jeu subtil sur le sens du verbe " guardare » est aussi celui qui protège son peuple, comme cela est exprimé le 25 décembre, dans G. Savonarola, Prediche sopra Aggeo, ouvr. cité, p. 364.

52. Ibid., p. 300.

53. Ibid., p. 428.

54. Ibid., p. 123-124.

55. Ibid., p. 125.

56. Ibid., p. 245-250.

57. Ibid., p. 325-326. Notons l'usage particulier du renvoi à la vision qui est fait ici, puisqu'on trouve, en quelques lignes, les formes suivantes du verbe vedere: «vedete », «non veggo », " io non lo veggo ", « io veggo", «non veggo", «veggo» (deux fois). Cette métaphore sera par la suite reprise, sous une forme plus élaborée stylistiquement, dans les Sermons sur les Psaumes, dans ce qui est sans doute le plus bel exemple d'hypotypose du discours savonarolien (G. Savonarola, Prediche sopra i Salmi, vol. 2, Rome, Belardetti, 1974, p. 179-180).

58. Cf. C. Ginzburg, «Ekphrasis et Connoisseurship », dans E. Alloa (éd.), Penser l'image, vol. III : Comment lire les images?, Dijon, Les Presses du Réel, 2017, p. 143.

59. G. Savonarola, Prediche sopra Aggeo, ouvr. cité, p. 196.

60. Ibid., p. 184-185.

61. Ibid., p. 170-171.

62. Ibid., p. 478.

63. G. Savonarola, Prediche sopra i Salmi, vol. 1, Rome, Angelo Belardetti, 1969, p. 204.

64. Bernardino da Siena, Le prediche volgari, vol.2, Pistoia, Pacinotti, 1934, p. 208 (cité dans O. Niccoli, Vedere, ouvr. cité, p.4). Les expressions «mentales oculi» et "mentis oculi» sont également présentes dans le douzième sermon (I, 2) du De sanctissimo Eucharistice Sacramento tel qu'il est reproduit dans l'édition d'Andrea Poletti (dans B. de Sienne, Sancti Bernardini Senensis Ordinis Seraphici Minorum Opera omnia, vol. 3, Venise, 1745, p. 66).

65. Cité dans L. Bolzoni, La rete delle immagini, ouvr. cité, p. 194. Une synthèse de l'usage médiéval de cette thématique nous est livrée dans B.Williamson, "Sensory Experience in Medieval Devotion: Sound and Vision, Invisibility and Silence», Speculum, vol. 88, $\mathrm{n}^{\circ} 1,2013, \mathrm{p} .12-14$.

66. Quintilien, Institution oratoire, Paris, Les Belles Lettres, 1978, t. 5, p. 77-78.

67. Cf. J.-P. Aygon, « "Les yeux de l'esprit” (oculi mentis, Quintilien, I. 0., 8, 3, 62) : la relation entre les images et la raison chez les rhéteurs et chez Sénèque », Pallas, $n^{\circ} 93,2013, \S 30$.

68. Cf. M. Miles, «The Eye of the Body and the Eye of the Mind in Saint Augustine's De trinitate and Confessions", The Journal of Religion, vol. 63, $\mathrm{n}^{\circ}$ 2, 1983, p. 131-134.

69. G. Savonarola, Prediche sopra Aggeo, ouvr. cité, p. 53.

70. Ibid., p. 39.

71. Ibid., p. 48-49.

72. "Vedi quello che io ti dico", dans G. Savonarola, Prediche sopra i Salmi, ouvr. cité, vol.1, p. 203-204.

73. G. Savonarola, Prediche sopra Aggeo, ouvr. cité, p. 307-308. 
74. Cf. R. Nelson, « Descarte's Cow and Other Domestications of the Visual », dans R. Nelson (dir.), Visuality before and beyond the Renaissance. Seeing as Others Saw, Cambridge, Cambridge University Press, 2000, p. 4-6.

75. Cf. H. Bredekamp, Théorie de l'acte d'image, Paris, Éditions de la Découverte, 2015, p. 181.

76. «Io ho tanto gridato e tanto vociferato, che io non so più che mi dire. » (G. Savonarola, Prediche sopra Aggeo, ouvr. cité, p. 11.)

77. W. J. T. Mitchell, «Que veulent réellement les images?», dans E. Alloa (éd.), Penser l'image, Dijon, Les Presses du Réel, 2010 et 2011, p. 239.

78. On perçoit distinctement ce sentiment d'incompréhension à la lecture du dernier texte que rédige Savonarole, le commentaire du psaume Miserere mei, dans G. Savonarola, Commento al salmo Miserere mei, Deus, Milan, Glossa, 2012.

\section{RÉSUMÉS}

Dans ses sermons consacrés au cycle d'Aggée ( $1^{\mathrm{er}}$ novembre-28 décembre 1494), Savonarole amène ses fidèles, par un processus de visualisation, à ouvrir l'« œil de leur esprit ", afin qu'ils aient accès au monde céleste et puissent atteindre la connaissance divine. Un second transfert, relevant d'un itinéraire spirituel plus complexe, les mène ensuite du champ doctrinal vers le champ politique, ouvrant ainsi sur un discours d'autorité. Si l'efficace du discours savonarolien doit beaucoup à ce double glissement, nous pouvons toutefois forger l'hypothèse que ce dernier porte également en lui les sources d'une possible déconnexion avec les fidèles, expliquant ainsi la rapidité de la chute finale du dominicain.

Nelle sue prediche dedicate al ciclo di Aggeo ( $1^{\circ}$ novembre-28 dicembre 1494), Savonarola porta i suoi seguaci, attraverso un processo di visualizzazione, ad aprire «l'occhio della loro mente», sicché possano accedere al mondo celeste e raggiungere la conoscenza divina. Un secondo spostamento, sorto da un itinerario spirituale più complesso, li porta poi dal campo dottrinale a quello politico, dando così vita ad un discorso di autorità. Se l'efficacia del discorso savonaroliano scaturisce da questo doppio spostamento, questo porta comunque in sé i semi di una possibile disconnessione con i fedeli, che in parte spiega la rapida caduta del Domenicano.

In his sermons devoted to the cycle of Haggai (1 November-28 December 1494), Savonarola leads his followers, through a process of visualization, to open the "eye of their mind", so that they have access to the celestial world and can get divine knowledge. A second transfer, which is part of a more complex spiritual journey, then takes them from the field of doctrine to the political field, thus opening up a discourse of authority. If the effectiveness of the savonarolian discourse owes much to this double shift, we can however make the assumption that it also carries the sources of a possible disconnection with the faithful, which explains in part the final fall of the Dominican. 
INDEX

Mots-clés : Savonarole, prédication, vision, « œil de l'esprit »

Keywords : Savonarola, preaching, vision, "eye of the mind"

Parole chiave : Savonarola, predica, visione, «occhio della mente»

\section{AUTEUR}

JEAN-MARC RIVIÈRE

Aix-Marseille Université, CAER, Aix-en-Provence, France.

jean-marc.riviere@univ-amu.fr 